\title{
Il rito inquieto. Storia dello yajña nell'India antica
}

Firenze, Società Editrice Fiorentina, 2018, pp. 370,

ISBN 978-88-6032-459-7, € 24,00

Duccio Lelli

\section{(2) OpenEdition}

\section{Journals}

Edizione digitale

URL: http://journals.openedition.org/mythos/372

DOI: $10.4000 /$ mythos.372

ISSN: 2037-7746

\section{Editore}

Salvatore Sciascia Editore

\section{Edizione cartacea}

Data di pubblicazione: 1 dicembre 2018

Paginazione: 155-158

ISBN: 978-88-8241-501-3

ISSN: $1972-2516$

Notizia bibliografica digitale

Duccio Lelli, « II rito inquieto. Storia dello yajña nell'India antica », Mythos [Online], 12 | 2018, online dal 24 septembre 2019, consultato il 23 septembre 2020. URL : http://journals.openedition.org/mythos/ 372 ; DOI : https://doi.org/10.4000/mythos.372 


\section{Marianna Ferrara}

II rito inquieto. Storia dello yajña nell'India antica

Firenze, Società Editrice Fiorentina, 2018, pp. 370, ISBN 978-88-6032-459-7, € 24,00.

\section{Duccio Lelli - ducciolelli@hotmail.it}

Il bel libro di Marianna Ferrara è il risultato maturo e completo di un'indagine in prospettiva storica sulla codifica della pratica rituale nell'India antica, avviata dall'Autrice durante un Dottorato di Ricerca presso I'Università Sapienza di Roma. Gli esiti di questa ricerca, già parzialmente divulgati in una monografia e in una serie di articoli apparsi fra il 2013 e il 2016, appaiono adesso rivalutati e sistemati in una cornice interpretativa coerente.

I motivi che hanno ispirato la ricerca sono insiti in un paradosso ben noto agli Indologi: sebbene lo yajña, il rito per eccellenza in ambito brahmanico, sia centrale nella sistemica del pensiero religioso indiano, in nessun testo ne viene mai data una definizione chiara e univoca, svincolata da metafore o tautologie. Fin dai testi più antichi è fiorita intorno allo yajña un'esegesi spasmodicamente minuziosa - al punto che, di molti rituali, noi conosciamo non solo contesto, modi e tempi, ma anche dettagli sulle figure professionali preposte, sull'ordine delle azioni e delle formule impiegate, sugli utensili, sulle materie oblative ecc. -, ma questa presenza ingombrante della pratica e la mole straordinaria dei particolari che l'accompagnano ne hanno sempre oscurato l'autentica natura storica. Si deve dunque accogliere con entusiasmo il coraggioso tentativo dell'Autrice che, a partire dai testi, ha cercato proprio questa natura così sfuggevole e trovatala, infine, nascosta fra le pieghe delle parole, ne ha tracciato la storia tormentata, portando alla luce una vicenda del tutto inedita.

Uno dei meriti maggiori del libro risiede appunto nel privilegiare i testi come fonti prioritarie delle ipotesi ermeneutiche e nell'indagarli a più livelli, oltre il dato meramente descrittivo; I'Autrice non si limita a riflettere su "cosa" i testi dicono, ma li interroga anche sul "quando" e sul "perché" lo dicono, cercando di cogliere le complesse dinamiche degli atti comunicativi di cui essi sono i veicoli. Impresa non facile, dal momento che i testi presi in esame coprono un arco cronologico di quasi due millenni e sono diversissimi per lingua (vedico, sanscrito classico, pāli, ardhamaghadi) tipologia (inni, trattati teologici e dottrinali, poemi epici, codici normativi, epigrafi) e stile (poesia, prosimetro, prosa narrativa, descrittiva, prescrittiva). La sfida linguistica è complicata dalla notevole difficoltà di stabilire la cronologia relativa dei testi e la loro dimensione autoriale; due problemi che l'Autrice affronta in modo originale, da una parte discutendo criticamente $\mathrm{i}$ dati di una ricchissima bibliografia, dall'altra eleggendo la storia della codifica dello yajña a elemento di prova capace di contribuire al dibattito. Lo studio etimologico e semantico, valido strumento dell'analisi filologica, permette inoltre di ridefinire il significato autentico di alcuni concetti chiave connessi alla pratica rituale.

Privilegiare un serio approccio testuale è già di per sé una scelta indicativa; si dovrà inoltre apprezzare anche l'attenzione costante riservata alle questioni teoriche e metodologiche, mai accettate aprioristicamente ma vagliate e selezionate con cura. Grazie a tale prospettiva storico-critica metodologicamente fondata e all'impegno filologico, l'Autrice è in grado di elaborare una nuova interpretazione dello yajña, che consiste nel considerarlo non un istituto codificato sempre uguale a sé stesso, espressione di una classe sacerdotale organizzata e solidale (come talvolta in passato si è pur sostenuto), bensì una pratica instabile, scossa da in- 
certezze, tensioni e antagonismi fra le parti in causa, continuamente ripensata e riqualificata anche su sollecitazione del mutato contesto storico: un rito, dunque, "inquieto".

II volume si articola in quattro capitoli, che "illustrano altrettanti snodi narratologici del discorso sulla nozione di yajña e mettono in primo piano gli interessi che percorrono le variazioni del discorso" (p. 17). II primo capitolo (L'assenza di quiete intorno allo yajña) affronta il problema dell'instabilità della pratica nel Rigveda, il più antico testo vedico. La difficoltà di stabilire una cronologia interna sicura non impedisce di cogliere ovunque, in questa raccolta di inni, la precarietà della procedura rituale, ancora tecnicamente incerta e piuttosto astratta, immersa in una dimensione di conflittualità endemica. Cominciando a pronunciarsi sulla pratica e sui modi di ottenerne l'efficacia, le élites di bhrāhmaña tradiscono uno stato di necessità urgente, riflesso nella preoccupazione di stabilire l'identità fra pratica e praticante, chiarire i ruoli dei diversi officianti del rituale e guadagnarsi il favore di ricchi e influenti patrocinatori. A questo proposito, per dimostrare che anche l'aspetto sintatticoterminologico illustra il "farsi" della pratica e chiarisce il linguaggio agonistico con cui $i$ praticanti hanno tentato di renderla modalità esclusiva per onorare gli dei, l'Autrice espone i risultati di una ricerca statistica sulla distribuzione delle occorrenze del tema nominale yajña- e della coniugazione della radice verbale yaj nel Rigveda. Uno studio sintattico-morfologico della radice e dei suoi derivati completa l'analisi linguistica. La maggior frequenza dei termini notevoli negli inni recenti della raccolta, l'articolazione sempre più variegata degli impieghi sintattici della radice e l'incremento di neoformazioni derivate e composti nominali e verbali svelano una strategia mirata all'istituzione del regolamento della pratica. In chiusura del capitolo, l'Autrice propone la lettura e il commento dell'inno quindicesimo dell'ottavo libro dell'Atharvaveda Paippalāda. Al plauso per aver incluso nella trattazione un testo così importante, che sebbene abbia finalmente guadagnato la meritata attenzione resta comunque abbastanza negletto oltre i confini della filologia vedica, si unisce la soddisfazione di veder confermato quanto la recensione Paippalāda dell'Atharvaveda sia un vero e proprio scrigno di tesori: in questo inno originalissimo, un bhrāhmaṇa che ha subito un'offesa chiama a raccolta bhrāhmaṇa di altri lignaggi, invitandoli a schierarsi uniti contro chi lo ha danneggiato, presumibilmente uno kșatriya laico. L'offesa, un danno contingente, diventa il pretesto per suggerire nuove sfumature relazionali fra officiante e patrocinatore: la cerchia di bhrāhmaṇa ci appare ancora insidiata da conflitti interni, ma allo stesso tempo confortata dalla possibilità di provvisorie coalizioni, strette in nome di una solidarietà fondata sull'orgogliosa consapevolezza della propria identità.

II secondo capitolo (La quiete apparente: come la pratica acquieta gli antagonismi) espone i tratti salienti di una fase avanzata della codifica dello yajña, quale emerge dalle raccolte dello Yajurveda e da alcune sezioni di Bhrāhmaṇa e Śrautasūtra: la codifica della pratica ha ricevuto una decisa accelerazione dal consolidamento del vincolo (anche economico) fra officianti e committenti e dal ricco corredo di istruzioni e direttive in merito alle azioni rituali. II linguaggio normativo ha stabilizzato e legittimato le prescrizioni in uno stato di arbitrarietà totale, con l'effetto di creare una quiete apparente intorno alla pratica. Apparente perché la formazione di entità politiche complesse ad opera di gruppi familiari ha accentuato le differenze procedurali fra lignaggi, inasprendo conflitti latenti o già manifesti, e all'interno dell'ambiente brahmanico emergono discrepanze nella forma di dilemmi sulle modalità rituali, sulla replicabilità dei riti e sul loro significato teologico. D'altro canto, mentre cresce l'apertura al confronto dialettico, si fa strada l'idea diffusa di dover preservare la pratica dall'ascolto non autorizzato. Di questa preoccupazione sono espressione eloquente gli Āranyaka, testi che circoscrivono la divulgazione del sapere rituale e costruiscono "una 
fortezza semiotica intorno alla trasmissione dei modi di compiere lo yajña" (p. 201), promuovendo un sapere a tratti esoterico, in cui l'attività rituale diventa quasi accessoria e decisamente secondaria rispetto alla conoscenza del significato nascosto e simbolico delle parole.

Con il terzo capitolo (La quiete tradita) la codifica dello yajña giunge a una svolta, che coincide con un periodo di cesura nella storia del pensiero indiano. I grandi cambiamenti socioeconomici avvenuti nelle regioni settentrionali dell'India a partire dal VI sec. a.C. sollecitano una radicale rivisitazione dell'istituto dello yajña: nuovi modi di pensare la pratica vengono elaborati sia da bhrāhmaña tradizionalisti sia da bhrāhmaṇa sensibili a nuove forme di spiritualità. Testi come gli Śrautasūtra, opere aforistiche dedicate esclusivamente alle forme di yajña delle occasioni pubbliche solenni, sono la voce di chi, aggrappandosi all'autorità della vecchia codifica, propone una riqualificazione nel solco della tradizione, attraverso l'attenzione all'atto rituale, la descrizione abbreviata di procedure semplificate e l'applicazione di facili tecniche mnemoniche. Le Upanișad, invece, pur ponendosi, almeno parzialmente, in continuità con la vecchia codifica, testimoniano il meraviglioso tentativo di riqualificare la pratica rendendola permeabile a nuove istanze. In polemica contro la falsa sapienza degli antichi, i bhrāhmaña che sono venuti a contatto con il fenomeno ascetico promuovono il vegetarianismo, invitano ad astenersi dall'uccisione di esseri viventi e riqualificano lo yajña come esercizio interiore, proponendo modalità esecutive indipendenti dall'uso di strumenti rituali ed estranee a complesse manipolazioni verbali e materiali. II concetto di azione rituale (karman) viene risemantizzato e acquista il significato di azione retributiva; lo yajña, definitivamente scisso dalla dimensione tecnica, si configura come pratica che inizia e finisce nel sé (ātman) e si realizza come conoscenza superiore (jñāna). Questa faticosa conquista personale si ottiene comunque ancora attraverso l'incontro-scontro in una dimensione pubblica che le Upanișad salvaguardano attentamente, nei dibattiti teologici e filosofici che emergono dai brahmodya e sono la spia di un agonismo sempre operante a livello della codifica della pratica.

Il quarto capitolo (Di nuovo senza quiete) si occupa di un periodo lungo e complesso, che si estende dal IV sec. a.C. fino a tutto il primo millennio dell'èra volgare. Le maggiori criticità che si riscontrano nella trasmissione della codifica dello yajña sono innescate dalle polemiche di buddhisti e jaina. Senza disprezzo, ma con toni di fermo e compassionevole ammonimento, le prime comunità buddhiste descrivono lo yajña come un sistema di relazioni economiche e politiche grazie alle quali avidi e ciechi bhrāhmaṇa si sono conquistati posizioni di prestigio, e come mezzo di accumulazione e violazione del dharma per eccellenza: i bhrāhmaña ottengono senza dare, uccidono esseri viventi e diffondono falsità, rivelando tutto il proprio decadimento morale nell'attaccamento ai beni materiali e nella debolezza di fronte alle passioni umane. Perentorie, pur nella diversità di premesse e toni, anche le critiche dei jaina, che non sfidano dialetticamente $\mathrm{i}$ bhrāhmaña, ma si limitano a rilevarne limiti ed errori: uccisori di animali, incapaci di controllare le facoltà sensoriali, privi di virtù, orgogliosi e sciocchi, non si accorgono che lo yajña è una delle cause di accumulo di karman, e come tale impedisce di intraprendere la via della liberazione.

In questo contesto problematico, emerge luminosa la figura del grande imperatore Aśoka. La sua vicenda, riletta con uno spirito critico che sappia rinunciare definitivamente al buonismo della tradizione agiografica, colpisce per il geniale pragmatismo con il quale il sovrano seppe ricoprire il ruolo di super partes fra i membri delle comunità sparse nel regno e di coagulante politico fra i vari gruppi sociali, la cui conflittualità fu contenuta grazie a principi di diffusa tolleranza. I suoi celebri editti su roccia, fatti sistemare strategicamente nei territori del regno, sono importanti anche per la storia dello yajña: diffondendo il precetto di non uccidere, essi 
hanno il significato di una presa di posizione decisa nei confronti dei gruppi brahmanici, non esplicitamente attaccati, ma di fatto penalizzati nel ruolo di officianti in cerimonie pubbliche.

Ormai vacillante sotto i colpi di critiche così autorevoli, la pratica dello yajña fu nuovamente riqualificata, con strategie differenti, entro le cerchie brahmaniche. Gli autori dei Dharmasūtra e Dharmaśāstra tentano di recuperare la codifica tradizionale, valorizzandone in pieno la dimensione tecnica e iscrivendola nel registro delle norme eticocomportamentali di una comunità linguisticamente compatta, religiosamente connotata e geograficamente circoscritta. Un nome su tutti, Manu: nella sua opera egli, "conservatore fra i conservatori” (p. 319), impone lo yajña quale norma comportamentale inseparabile dai canoni tradizionali dell'audizione autorizzata, mantenendo uno strategico silenzio sulla conflittualità insita nella pratica. La Bhagavadgīta è invece espressione di un disegno che orienta il ripristino della vecchia codifica verso nuovi orizzonti culturali e filosofici. La supremazia dello yajña viene stabilita ricorrendo a un'etica analoga a quella dei movimenti ascetici (divieto di uccidere, vegetarianesimo) e sviluppando gli insegnamenti upanișadici sull'azione retributiva e disinteressata.

A testimonianza di un'instabilità davvero connaturata alla pratica, il volume si chiude con un breve cenno alla riflessione del movimento mimāṃsāka, che durante tutto il primo millennio dell'èra volgare, ripartendo dal punto precedente alla rottura dell'assetto interno, si propone il ripristino delle coordinate del dharma brahmanico, caratterizzato da azioni e prescrizioni rituali. Tracciando la parabola dello yajña, l'Autrice ne ha così recuperato la natura storica: concetto multiforme e camaleontico, valvola di sfogo delle ansie di epoche diverse, arena di scontro fra sacerdoti e moneta di scambio fra officianti e committenti, oggetto di dibattiti e critiche più o meno feroci, fucina d'idee e catalizzatore di nuove esperienze spirituali, lo yajña è davvero un rito la cui congenita inquietudine si conferma paradigma interpretativo valido e originale. Senza dubbio, un libro così pregevole meriterebbe una ristampa che eliminasse le rare imprecisioni di traduzione e i numerosi refusi. 